\title{
Importancia de los estilos de socialización parental en los roles del acoso entre pares
}

\author{
Importance of the parental socialization style
}

in the roles of harassment among peers

\author{
José Hernando Ávila-Toscano ${ }^{1}$, Eliana Álvarez Fontalvo², \\ Laura Isabel Rambal-Rivaldo ${ }^{3}$ y Leonardo Vargas-Delgado ${ }^{4}$
}

\begin{abstract}
${ }^{1}$ Psicólogo. Magíster en Psicología. Doctor en Ciencias Humanas y Sociales. Líder científico en el grupo de investigación PSICUS (Psicología, Cultura y Sociedad) de la Corporación

Universitaria Reformada (Colombia). E-mail: javila@unireformada.edu.co

${ }^{2}$ Psicóloga. Magíster en Educación. Investigadora adscripta al grupo de investigación PSICUS (Psicología, Cultura y Sociedad) de la Corporación Universitaria Reformada (Colombia).

E-mail: elina.alvarez.g@gmail.com

${ }^{3}$ Psicóloga. Investigadora adscripta al grupo de investigación PSICUS (Psicología, Cultura y

Sociedad) de la Corporación Universitaria Reformada (Colombia).

E-mail: laura.rambal@unireformada.edu.co

${ }^{4}$ Licenciado en Matemáticas. Magíster en Educación. Coordinador del pregrado de Licenciatura en Matemáticas de la Universidad del Atlántico. Investigador adscripto al grupo de investigación

GIMED (Colombia). E-mail: ljvargas@mail.uniatlantico.edu.co
\end{abstract}

Corporación Universitaria Reformada, Universidad del Atlántico.

Barranquilla, Colombia.

\section{Resumen}

La familia es un escenario primordial en el estudio del acoso entre iguales, y particularmente la relación paterno-filial ha cobrado relevancia en la investigación científica sobre el tema. En este sentido, la socialización parental puede ser clave como factor de protección o de riesgo en la aparición de situaciones de acoso. El presente estudio aborda el análisis de estrategias y estilos de socialización de padres y madres en estudiantes de secundaria implicados en acoso escolar, como testigo, agresor, víctima y agresor-víctima. Se evaluaron 551 adolescentes de entre 12 y 18 años mediante un diseño comparativo de grupos naturales. Se aplicó el cuestionario del defensor del pueblo sobre violencia escolar ajustado para contexto colombiano y la escala de estilos de socialización parental en la adolescencia (ESPA 29). Como resultado se identificaron en los participantes altos niveles de estrategias de socialización basadas en coerción/imposición por parte de ambas figuras paternas. A diferencia de estudios previos, no se identifica relación entre ninguno de los estilos parentales de la madre y los roles asumidos por los estudiantes en situaciones de acoso, mientras que el estilo indulgente en el padre mostró relación estadísticamente significativa con los roles de testigo y agresor-víctima. Se discuten las implicaciones de los resultados resaltando la importancia de la crianza afectiva y el rol que desempeñan las figuras parentales en la socialización y formación de habilidades psicosociales que contri- 
buyan en la disminución de las prácticas de acoso en niños y adolescentes.

Palabras clave: socialización parental, roles acoso escolar, adolescentes

\section{Abstract}

The family is a primary scenario when studying harassment among equals. Particularly, the parent-child relationship has built up importance in scientific investigations regarding this topic. In this regard, parental socialization may be key as a protection or risk aspect in the occurrence of harassment situations.

The development of this study focuses in the achievement of two main objectives. The first objective is aimed to identifying differences in the strategies of parental socialization in both parents, in accordance to the role of the participants involved in harassing behavior. The second objective is based in stablishing if the roles adopted by students have any co-relation with the parental socialization style.

In order to fulfill these objectives an empirical, comparative, investigation took place that was based in the design of natural groups, which facilitates the comparison of different variables in social groups that belong to a same culture, e.g. students, where the analyzed variables source differences among individuals. The study had a base of 551 students (290 male and 261 female) from four different educational institutions, with ages ranging from 12 to $18(\mu=15.2$, of $=1.3)$. Harassment conducts were evaluated in accordance to the Defensor del Pueblo questionnaire, from a version adjusted to the Colombian context. Whereas the parental style of socialization was evaluated in accordance with Escala de Estilos de Socialización Parental en la Adolescencia (ESPA-29).

The participant-type descriptive evaluation highlights that $296(53.8 \%)$ of the students take part as bully-victims, $95(17.2 \%)$ take part as non-involved, $87(15.8 \%)$ take part as victims, and $73(13.2 \%)$ take part as bullies. These roles were embraced similarly in men and women without a significant relationship between gender and the type of participant $\left(\chi^{2}\right.$ $=5.328, p=.149>.05)$.

Concerning to the socialization strategies of parental figures, in the mother's case, the obtained data indicates that the main differences are found in the group of students who embrace the role of offender-victims. In this group, participants show a higher level of indifference that non-involved $(p=.023<.05)$ and bullies $(p=.007<.05)$, a higher level of physical coercion than non-involved ( $p=.000$ $<.05)$ and victims $(p=.023<.05)$, as well as a higher level of the strictness/imposition axis than non-involved $(p=.001<.05)$. The latest show higher verbal coercion $(p=.009<.05)$ and strictness/imposition $(p=.032<.05)$ than bullies.

In the father's case, the obtained data also highlights the bully-victims as the group with higher ennui than non-involved $(p=.000<$ $.05)$ and bullies $(p=.046<.05)$, higher indifference than non-involved $(p=.000<.05)$ and bullies $(p=.010<.05)$, higher physical coercion than non-involved $(p=.000<.05)$ and victims $(p=.021<.05)$, as well as a higher number than non-involved $(p=.001<$ $.05)$ in the strictness/imposition axis.

The most common parental style strategy in mothers is indulgent $(\mathrm{n}=240,43.6 \%)$, followed by authoritative $(\mathrm{n}=161,29.2 \%)$, neglectful $(\mathrm{n}=102,18.5 \%)$ and last authori$\operatorname{tarian}(\mathrm{n}=48,8.7 \%)$. Meanwhile, the indulgent style is also the most common among fathers $(n=211,38.3 \%)$, followed by neglectful $(\mathrm{n}=171,31 \%)$, authoritative $(\mathrm{n}=109$, $19.8 \%)$ and authoritarian $(\mathrm{n}=60,10.9 \%)$. This discards the existence of a significant association between the mother's socialization style and student's harassment roles. On the other hand, the indulgent style in fathers was significant in relation to the witnesses $\left(\chi^{2}\right.$ $=16.88, p=.000>.001 ; \varpi=.18,1-\beta=.97)$ and the bully-victims $\left(\chi^{2}=8.96, p=.03>.05\right.$; $\varpi=.14,1-\beta=.80$ ).

The parenthood focused on fulfilling the children demands with low boundaries deli- 
mitation is reiterative in parents where indulgent is their most common style of parent socialization, which seems to be associated with an expectation to harass.

This work highlights the important role that the parent figures play in the formation of psychosocial skills to the integration of children and teenagers. Furthermore, it features the importance of how parents build bonding relationships with their children.

Keywords: parental socialization, harassment roles, teenagers

\section{Introducción}

La literatura científica coincide en señalar que el acoso entre iguales (bullying) es un fenómeno que implica conductas agresivas de índole física, verbal, psicológica, social (Landazabal y Oñederra, 2010) e incluso mediadas por la tecnología (Charalampous et al., 2018), las cuales son ejercidas por un estudiante, o grupo de estos, de forma repetitiva y sistemática contra compañeros generalmente más débiles, quienes pasan a ser las víctimas habituales de tales acciones (Cerezo, 2010). Dichas conductas se ejecutan con la intención primordial de causar daño (Enríquez y Garzón, 2015).

En la situación de acoso, los actores implicados pueden presentar distintos roles según sean objeto de las agresiones, testigos de estas o quienes las provocan. En este sentido, se describe a tales actores como agresores, víctimas, observadores o testigos $\mathrm{y}$ agresor-víctima (Del Rey y Ortega, 2008; Smith, Morita, Junger-Tass, Catalana y Slee, 1999); cada uno de ellos posee rasgos y características particulares (Resett, 2018) y contribuye de manera relevante al círculo de maltrato $\mathrm{y}$, al mismo tiempo, resultan influenciados negativamente por múltiples factores de naturaleza social, personal, educativa y familiar (Buelga, Cava y Musitu, 2012; Cerezo y Ato, 2010; Rigby, 2005).

El presente trabajo se centra en el factor familiar como relevante en los escolares, ya que actúa como elemento de protección o riesgo frente a situaciones de violencia entre iguales (León del Barco, Felipe-Castaño, Polo del Río y Fajardo-Bullón, 2015). Distintos estudios han jerarquizado el rol de la socialización parental y los estilos de crianza que evidencian el papel que las prácticas, las actitudes y los comportamientos parentales tienen en relación con las conductas agresivas de los hijos en la escuela (Georgiou, Stavrinides y Fousiani, 2013; Georgiou, Ioannou y Stavrinides, 2018).

Los estilos de socialización parental describen cómo perciben los hijos a sus padres y sus prácticas de socialización (Baumrind, 1991; Maccoby y Martin, 1983). La interacción de los padres con sus hijos incluye la forma en que responden a sus necesidades (responsividad) y la manera en que utilizan el control (exigencia) (Charalampous et al., 2018), dimensiones que definen las diversas actitudes, los valores, las prácticas y los comportamientos de los padres en los diferentes estilos parentales (García y Gracia, 2010; Musitu y García, 2004).

La literatura científica sobre el tema es diversa, pues en el caso de progenitores con estilo negligente se ha indicado que este tipo de interacción, mediada por la falta de afecto y ausencia de control, no favorece el ajuste personal y social de los hijos, pudiendo provocar conductas socialmente inadecuadas como la agresividad hacia los iguales (LópezSoler, Puerto, López-Pina y Prieto 2009). Asimismo, entre los agresores se ha identificado que perciben negativamente la confianza y comunicación con sus figuras parentales (Simões, Ferreira, Braga y Testa, 2015).

Estudios recientes señalan que los agresores y las víctimas tienen progenitores con mayores niveles de estrés parental, que utilizan prácticas educativas autoritarias o punitivas (Gómez-Ortiz, Romera y Ortega-Ruiz, 2016) $\mathrm{y}$ en algunas ocasiones tienden a ser permisivos. Además, los padres de los agresores presentan menor nivel de competencia parental (Garaigordobil y Machimbarrena, 2017). Otras 
investigaciones, como la de Pérez y Castañeda (2015), coinciden en que el papel de agresor se asocia con un estilo paterno autoritario y con madres permisivas, al tiempo que plantean que la victimización es común en hijos de padres permisivos y madres democráticas.

El estudio de Charalampous et al. (2018) refuerza parte de esta evidencia. Estos autores evaluaron una muestra de 861 niños y adolescentes chipriotas entre quienes hallaron que la intimidación se asocia con el estilo parental autoritario. Los progenitores que aplican este estilo tienden a usar prácticas punitivas $\mathrm{y}$ aceptan la violencia como mecanismo para solucionar los conflictos. Los hijos, por su parte, perciben a sus padres como insensibles a sus propios problemas, lo que no les permite el desarrollo de la empatía y utilizan la desigualdad y la competencia como una estrategia interpersonal, mostrándose a la sociedad como agresivos. De acuerdo con Charalampous et al. (2018), estos jóvenes también se exponen a ser acosados, de manera que pueden asumir el rol mixto de agresor-víctima. Según estos autores, los padres permisivos también ejercen un efecto sobre la victimización, dado que suelen ser sobreprotectores, lo que pone en riesgo a sus hijos frente al acoso (Charalampous et al., 2018), resultado análogo a lo propuesto por otros trabajos (Garaigordobil y Machimbarrena, 2017; Pérez y Castañeda, 2015).

En general, la crianza permisiva se relaciona con una mayor tendencia a la soledad por parte de los adolescentes (Palomar y Victorio, 2018) y son diversas las investigaciones que recalcan el papel de la sobreprotección y permisividad en la victimización, especialmente por parte de la figura materna (Cerezo, Sánchez, Ruiz y Arense, 2015). Al parecer, las madres permisivas presentan una alta capacidad de respuesta (responsividad) hacia las necesidades de sus hijos, lo que hace que se desborden en cuidados y protección, situación que parece predisponer a los hijos a sufrir situaciones de acoso, por encima de los hijos de madres con otro estilo de socialización (Georgiou, 2008).
Respecto al estilo autorizativo, se ha observado que progenitores que practican el diálogo, control parental moderado y expresión afectiva, permiten al adolescente identificar los límites establecidos por los adultos sin necesidad de ser autoritarios (GómezOrtiz, Del Rey, Casas y Ortega, 2014), además de facilitarles contar con mejores niveles de adaptación social (Parra y Oliva, 2006). Sin embargo, no hay conclusiones claras respecto a dicho estilo, pues otras investigaciones han referenciado que las víctimas también pueden contar con madres tanto autoritativas como democráticas (Sánchez, 2009), e incluso este estilo puede identificarse en porcentajes similares en casos de estudiantes victimizados con padres autoritarios (Cerezo et al., 2015).

Tal y como señalan Cerezo et al. (2015), no existe plena confirmación acerca de un estilo de socialización parental asociado con la intimidación o acoso, si bien las prácticas autoritarias parecen más comunes entre agresores y la permisividad entre las víctimas. Al ser las formas de socialización parental un fenómeno de mucha relevancia por el papel sustancial del entorno familiar y puntualmente de la crianza de los padres sobre el desarrollo (Garaigordobil y Machimbarrena, 2017), este estudio apunta a contribuir al tema en discusión, y para ello se plantean los siguientes objetivos: (a) identificar diferencias en las estrategias de socialización parental en ambos progenitores de acuerdo con el rol de los participantes implicados en conductas de acoso; y (b) establecer si los roles asumidos en conductas de acoso por estudiantes, se relacionan con los estilos de socialización parental.

\section{Método}

\section{Diseño}

Se desarrolló una investigación empírica, comparativa, basada en un diseño de grupos naturales (Ato, López y Benavente, 2013), que permite la comparación de diversas variables dependientes en grupos preexistentes que pertenecen a una misma cultura (por ejemplo, estu- 
diantes) y entre los cuales se observan diversos niveles de variables que son fuente de diferencias entre los individuos (Ato et al., 2013).

\section{Participantes}

El estudio se realizó con 551 estudiantes de cuatro instituciones educativas de la ciudad de Barranquilla (Colombia), escogidos de forma intencional sin considerar su nivel de desempeño, sexo o antecedentes disciplinarios. Los evaluados contaban con edades comprendidas entre 12 y 18 años $(\mu=15.2$, de $=1.3)$, el $52.6 \%(n=290)$ eran hombres y el $47.4 \%$ $(n=261)$, mujeres. Según su formación, 22\% $(n=121)$ cursaba grado octavo, $29 \%(n=160)$ noveno, $25.4 \%(n=140)$ décimo y $23.6 \%$ $(n=130)$ undécimo.

\section{Instrumentos}

\section{Cuestionario del defensor del pueblo}

Este cuestionario fue diseñado por un grupo de investigadores de la Universidad Autónoma de Madrid para el Informe del Defensor del Pueblo sobre violencia escolar (2007). Se basa en otras pruebas a las cuales se les hizo adaptaciones específicas. El instrumento ha sido ajustado para el contexto colombiano (Hoyos, Aparicio y Córdoba, 2005) y está formado por tres secciones principales que permiten identificar acoso observado, realizado y experimentado, describiendo los diferentes tipos de maltrato y la manera como se dan a través de escala tipo Likert de 4 puntos (desde 0: Nunca, hasta 3: Siempre). La prueba ha sido usada exitosamente en estudiantes del Caribe colombiano (Ávila-Toscano, Marenco-Escuderos y Tilano, 2014).

\section{Escala de estilos de socialización parental en la adolescencia (ESPA-29) (Musitu y García, 2001)}

Es un instrumento que consta de 29 ítems idénticos para dos subescalas, la primera aplica a la identificación de los estilos de socialización de la madre y la segunda se dirige a identificarlos en el padre. Todos los reactivos cuentan con una escala tipo Likert desde 1 (nunca) hasta 4 (siempre). El instrumento identifica dos ejes de la socialización que integran siete estrategias parentales; de este modo permite reconocer el eje I denominado "Aceptación/Implicación", que incluye estrategias como afecto, diálogo, indiferencia y displicencia; mientras que el eje II evalúa "Coerción/Imposición", que incluye las estrategias privación, coerción verbal y coerción física. La medición de estas características permite identificar cuatro estilos parentales ajustados a la teoría propuesta por los autores (autoritario, autorizativo, indulgente, negligente). La obtención de las puntuaciones definitivas del instrumento se realiza mediante tablas de baremos ajustadas según edad y sexo del estudiante, y también según el progenitor evaluado (padre/madre). El instrumento ha sido empleado con población colombiana con buenos resultados (Andrade y Gonz, 2017; Salamanca-Ramos, Chávez-Ávila y Carmona-Parra, 2017).

\section{Procedimiento}

El acceso a la población se logró gracias al establecimiento de contactos formales con el cuerpo directivo de las instituciones educativas. Tras socializar el objetivo y alcance del estudio se obtuvo autorización para comunicar el propósito de la investigación a los padres de los estudiantes, quienes autorizaron la participación de los menores, y estos, por su parte, informaron su consentimiento.

La aplicación de los instrumentos se hizo de forma grupal, en jornadas únicas aprovechando la presencia del estudiantado en las actividades académicas. Todo el proceso estuvo supervisado por docentes y autoridades de cada institución educativa. La información recogida fue analizada a través de procedimientos no paramétricos dado que los datos no cumplieron el supuesto de normalidad. El 
análisis se basó en la comparación de grupos independientes con la $H$ de Kruskal-Wallis, definiendo como grupos los diferentes roles asumidos por lo estudiantes en las conductas de maltrato, para comparar si presentaban diferencias significativas en relación con las estrategias de socialización parental.

En todos los casos en que se hallaron diferencias significativas, se cumplió el análisis post hoc con la $U$ de Mann-Whitney, calculando el tamaño de efecto con la $r$ de Rosenthal. Finalmente, se aplicó Chi cuadrado para calcular la existencia de asociación entre el estilo parental de ambos progenitores y el rol asumido por los estudiantes en las situaciones de acoso; el tamaño de efecto se calculó con el índice $\varpi$ y la identificación puntual de las categorías que expresan asociaciones se definió con el cálculo de los Residuos Tipificados Corregidos (RTC).

\section{Resultados}

En la evaluación descriptiva del tipo de actor destacaron los estudiantes que actúan en calidad de agresor-víctima $(n=296 ; 53.8 \%)$, seguidos en valores similares de los testigos $(n$ $=95 ; 17.2 \%)$ y las víctimas $(n=87 ; 15.8 \%)$, mientras que los actores de menor frecuencia fueron los agresores $(n=73 ; 13.2 \%)$. De acuerdo con el sexo, los hombres se identifican en mayor medida con el rol de agresor-víctima $(n=161 ; 55.5 \%)$ y como testigo
( $n=56 ; 19.3 \%)$, mientras que la identificación como víctima $(\mathrm{n}=38 ; 13.1 \%)$ y como agresor $(\mathrm{n}=35 ; 12.1 \%)$ tiene valores similares. Las mujeres, así como los varones, sobresalen en el rol de agresor-víctima ( $n=135,51.7 \%)$, seguido del rol de víctimas $(n=49,18.8 \%)$, y en valores similares se identificó el rol como testigo ( $n=39,14.6 \%)$ y agresor $(n=38,14.9$ $\%)$. Sin embargo, los resultados obtenidos no hallaron relación significativa entre el sexo de los estudiantes y el tipo de actor $\left(x^{2}{ }_{[3]}=5.328\right.$, $p=.149$ >.05), y tampoco hubo significación con el grado cursado $\left(x^{2}{ }_{[9]}=5.913, p=.749\right.$ $>$.05).

Respecto a la socialización, en la Tabla 1 se presentan los datos descriptivos de las estrategias empleadas por cada uno de los progenitores; asimismo, la tabla contiene los resultados del análisis posterior dirigido a encontrar diferencias significativas entre tales estrategias y el tipo de actor. Este procedimiento se cumplió aplicando el estadístico $H$ de Kruskal-Wallis lo que permitió identificar que, en cuanto a la madre, las significancias halladas correspondieron a las estrategias de Indiferencia, Coerción física y Coerción verbal, así como el eje de Coerción/Imposición, mientras que en relación con el padre las significancias halladas se dieron con las variables Displicencia, Indiferencia y Coerción física, y al igual que con la madre, destacó el eje Coerción/Imposición.

Tabla 1

Estadísticos descriptivos de las estrategias de socialización parental y prueba $\mathrm{H}$ de Kruskal-Wallis $\left(\chi^{2}\right)$.

\begin{tabular}{c|cccc|cccc}
\multirow{2}{*}{$\begin{array}{c}\text { Estrategia de socializa- } \\
\text { ción }\end{array}$} & \multicolumn{4}{|c|}{ Madre } & \multicolumn{4}{c}{ Padre } \\
\cline { 2 - 9 } & $\boldsymbol{M}$ & $\boldsymbol{D E}$ & $\chi^{2 a}$ & $\boldsymbol{p}$ & $\boldsymbol{M}$ & $\boldsymbol{D E}$ & $\chi^{2 a}$ & $\boldsymbol{p}$ \\
\hline Diálogo & 2.70 & .75 & 1.33 & .72 & 2.54 & .88 & .12 & .97 \\
Afecto & 3.35 & 10.0 & .32 & .95 & 2.70 & .95 & .69 & .87 \\
Displicencia & 1.39 & .52 & 5.13 & .16 & 1.72 & 4.58 & 16.06 & $.00^{*}$ \\
Indiferencia & 1.40 & .54 & 11.36 & $.01^{*}$ & 1.53 & .70 & 19.48 & $.00^{*}$
\end{tabular}




\begin{tabular}{c|cccc|cccc}
\multirow{2}{*}{$\begin{array}{c}\text { Estrategia de socializa- } \\
\text { ción }\end{array}$} & \multicolumn{4}{|c|}{ Madre } & \multicolumn{4}{c}{ Padre } \\
\cline { 2 - 9 } & $\boldsymbol{M}$ & $\boldsymbol{D E}$ & $\boldsymbol{\chi}^{2 \boldsymbol{a}}$ & $\boldsymbol{p}$ & $\boldsymbol{M}$ & $\boldsymbol{D E}$ & $\chi^{2 \boldsymbol{a}}$ & $\boldsymbol{p}$ \\
\hline Aceptación/Implicación & 3.19 & .48 & 3.42 & .35 & 3.04 & .59 & 5.18 & .15 \\
Coerción Física & 1.69 & .77 & 23.64 & $.00 *$ & 1.60 & .74 & 21.59 & $.00^{*}$ \\
Privación madre & 1.40 & .50 & 1.88 & .59 & 1.70 & 7.67 & 2.56 & .46 \\
Coerción Verbal & 1.97 & .68 & 11.36 & $.03 *$ & 1.85 & .70 & 1.32 & .72 \\
Coerción/Imposición & 1.26 & .80 & 8.09 & $.04 *$ & 1.22 & .79 & 12.85 & $.00 *$
\end{tabular}

${ }^{a}$ grados de libertad: $3 .{ }^{*} p<.05$. Variable de contraste: Tipo de actor.

Los contrastes post hoc se cumplieron con el estadístico U de Mann Whitney, cuyos resultados se aprecian en la Tabla 2 acompañados del tamaño de efecto ( $r$ de Rosenthal). Frente a las estrategias empleadas por la madre, los datos señalan que las principales diferencias se encuentran en el grupo de estudiantes identificados como agresores-víctimas. Este conjunto de actores presenta mayores niveles de indi- ferencia y de coerción verbal que testigos y agresores, mayor coerción física que testigos y víctimas, y también se observan mayores rangos medios del eje Coerción/Imposición que en los testigos. Estos últimos por su parte, presentan rangos medios más elevados de Coerción verbal y del eje Coerción/Imposición que los agresores.

Tabla 2

Análisis post hoc con prueba $U$ de Mann Whitney y su respectivo tamaño de efecto para las estrategias de socialización parental según tipo de actor.

Contrastes correspondientes a las estrategias de socialización materna

\begin{tabular}{cccccccc}
\hline \multirow{2}{*}{ Variable } & Grupo 1 & $\begin{array}{c}\text { Grupo } \\
\text { contraste }\end{array}$ & $\boldsymbol{U}$ & $\boldsymbol{Z}$ & $\boldsymbol{p}$ & $\boldsymbol{r}^{a}$ & $>\boldsymbol{R} \boldsymbol{M}$ \\
\hline \multirow{2}{*}{ Indiferencia } & $\mathrm{T}$ & $\mathrm{A}-\mathrm{V}$ & 11891.0 & -2.280 & $.023^{*}$ & $.11^{b}$ & $\mathrm{~A}-\mathrm{V}$ \\
& $\mathrm{A}$ & $\mathrm{A}-\mathrm{V}$ & 8630.0 & -2.685 & $.007^{*}$ & $.14^{b}$ & $\mathrm{AV}$ \\
\hline \multirow{3}{*}{ Coerción física } & $\mathrm{T}$ & $\mathrm{A}$ & 2419.5 & -3.269 & $.001^{*}$ & $.25^{b}$ & $\mathrm{~A}$ \\
\cline { 2 - 8 } & $\mathrm{T}$ & $\mathrm{A}-\mathrm{V}$ & 9704.5 & -4.575 & $.000^{*}$ & $.23^{b}$ & $\mathrm{~A}-\mathrm{V}$ \\
& $\mathrm{V}$ & $\mathrm{A}-\mathrm{V}$ & 10828.0 & -2.268 & $.023^{*}$ & $.12^{b}$ & $\mathrm{~A}-\mathrm{V}$ \\
\hline \multirow{2}{*}{ Coerción verbal } & $\mathrm{T}$ & $\mathrm{A}$ & 2655.0 & -2.602 & $.009^{*}$ & $.20^{b}$ & $\mathrm{~T}$ \\
& $\mathrm{~A}$ & $\mathrm{~A}-\mathrm{V}$ & 8900.0 & -2.334 & $.020^{*}$ & $.12^{b}$ & $\mathrm{~A}-\mathrm{V}$ \\
\hline \multirow{2}{*}{ Coerción / Imposición } & $\mathrm{T}$ & $\mathrm{A}$ & 2796.0 & -2.149 & $.032^{*}$ & $.17^{b}$ & $\mathrm{~T}$ \\
& $\mathrm{~T}$ & $\mathrm{~A}-\mathrm{V}$ & 11544.5 & -2.625 & $.009^{*}$ & $.13^{b}$ & $\mathrm{~A}-\mathrm{V}$ \\
\hline
\end{tabular}


Contrastes correspondientes a las estrategias de socialización paterna

\begin{tabular}{cccccccc}
\hline \multirow{2}{*}{ Variable } & Grupo 1 & $\begin{array}{c}\text { Grupo } \\
\text { contraste }\end{array}$ & $\boldsymbol{U}$ & $\boldsymbol{Z}$ & $\boldsymbol{p}$ & $\boldsymbol{r}^{\boldsymbol{a}}$ & $>\boldsymbol{R} \boldsymbol{M}$ \\
\hline \multirow{2}{*}{ Displicencia } & $\mathrm{T}$ & $\mathrm{A}-\mathrm{V}$ & 10590.0 & -3.662 & $.000^{*}$ & $.19^{b}$ & $\mathrm{~A}-\mathrm{V}$ \\
& $\mathrm{A}$ & $\mathrm{A}-\mathrm{V}$ & 9188.5 & -1.999 & $.046^{*}$ & $.10^{b}$ & $\mathrm{~A}-\mathrm{V}$ \\
\hline \multirow{3}{*}{ Indiferencia } & $\mathrm{T}$ & $\mathrm{A}$ & 3356.0 & -2.239 & $.025^{*}$ & $.17^{b}$ & $\mathrm{~T}$ \\
\cline { 2 - 8 } & $\mathrm{T}$ & $\mathrm{A}-\mathrm{V}$ & 10280.5 & -4.001 & $.000^{*}$ & $.20^{b}$ & $\mathrm{~A}-\mathrm{V}$ \\
& $\mathrm{A}$ & $\mathrm{A}-\mathrm{V}$ & 8714.0 & -2.590 & $.010^{*}$ & $.13^{b}$ & $\mathrm{~A}-\mathrm{V}$ \\
\hline \multirow{3}{*}{ Coerción física } & $\mathrm{T}$ & $\mathrm{A}$ & 2417.0 & -3.420 & $.001^{*}$ & $.26^{b}$ & $\mathrm{~A}$ \\
\cline { 2 - 8 } & $\mathrm{T}$ & $\mathrm{V}$ & 3229.0 & -2.545 & $.011^{*}$ & $.19^{b}$ & $\mathrm{~V}$ \\
\cline { 2 - 8 } & $\mathrm{T}$ & $\mathrm{A}-\mathrm{V}$ & 10052.5 & -4.215 & $.000^{*}$ & $.21^{b}$ & $\mathrm{~A}-\mathrm{V}$ \\
\multirow{2}{*}{ Coerción / Imposición } & $\mathrm{V}$ & $\mathrm{A}-\mathrm{V}$ & 10792.0 & -2.309 & $.021^{*}$ & $.12^{b}$ & $\mathrm{~A}-\mathrm{V}$ \\
\cline { 2 - 8 } & $\mathrm{T}$ & $\mathrm{A}$ & 2486.5 & -3.139 & $.002^{*}$ & $.24^{b}$ & $\mathrm{~A}$ \\
\cline { 2 - 8 } & $\mathrm{T}$ & $\mathrm{A}-\mathrm{V}$ & 11009.5 & -3.183 & $.001^{*}$ & $.16^{b}$ & $\mathrm{~A}-\mathrm{V}$
\end{tabular}

Notas: T: testigo; A: Agresor; V: Víctima; A-V: Agresor-víctima. $r^{a}: \mathrm{r}$ de Rosenthal (tamaño de efecto). ${ }^{b}$ efecto pequeño. $<R M$ : grupo con mayor rango medio. * $p<.05$.

En el caso de las estrategias implementadas por la figura paterna (ver Tabla 2), también sobresalen los agresores-víctimas como el grupo de actores con mayor registro de Displicencia e Indiferencia que testigos y agresores, mayor Coerción física que testigos y víctimas, así como valores más elevados en el eje Coerción/Imposición que los testigos. Entre las víctimas y los agresores, también destaca la Coerción física en comparación con los testigos y, en este último grupo se registra mayor Indiferencia en comparación con los agresores.

Ahora bien, los resultados de las diferentes estrategias y sus correspondientes ejes de socialización, facilitan identificar cuatro estilos parentales en cada progenitor. De esta forma, en este estudio se reporta que el estilo más común en la madre es el Indulgente ( $n=$ $240,43.6 \%$ ), seguido del estilo autorizativo $(n=161,29.2 \%)$, del negligente $(n=102$, $18.5 \%)$ y, por último, del autoritario $(n=48$, $8.7 \%$ ). El estilo indulgente se repitió como el más frecuente entre los padres $(n=211,38.3$ $\%)$, seguido del negligente $(n=171,31 \%)$, el autorizativo $(n=109,19.8 \%)$ y finalmente del autoritario $(n=60,10.9 \%)$. Al hacer el análisis para comprobar la relación entre dichos estilos y el tipo de actor en conductas de acoso, se descartó la existencia de significancia estadística frente al papel de la socialización materna, mientras que el estilo del padre fue significativo en relación con los testigos $(w=.18,1-\beta=.97)$ y los agresores víctimas $(w=.14,1-\beta=.80)$ (Tabla 3$)$. 
Tabla 3

Cálculo de asociación entre el estilo parental de ambos progenitores y el rol asumido en situaciones de acoso.

\begin{tabular}{c|cc|cc}
\multirow{2}{*}{ Rol } & \multicolumn{3}{c}{ Padre } & \multicolumn{2}{c}{ Madre } \\
\hline Testigo & 16.88 & $.00^{* * *}$ & 3.87 & .27 \\
Agresor & 1.48 & .68 & 1.40 & .70 \\
Víctima & 3.49 & .32 & 4.05 & .25 \\
Agresor-víctima & 8.96 & $.03^{*}$ & 2.24 & .52
\end{tabular}

${ }^{*} p<.05 ;{ }^{* * *} p<.001$

La socialización parental basada en el estilo indulgente fue la que sobresalió en el análisis realizado. De este modo, se relaciona con el rol de testigo de forma positiva $(\mathrm{RTC}=$ 4.1). En cambio, frente al rol de agresor-víctima la frecuencia es inferior a la teórica, por lo que se asume que la relación es negativa $(\mathrm{RTC}=-2.9)$.

\section{Discusión}

Dos objetivos orientaron el desarrollo de este estudio, frente al primero, enfocado en identificar diferencias en las estrategias de socialización parental, de acuerdo con el rol de los participantes, se diferencian los hallazgos relevantes de acuerdo con el papel de la madre y el padre.

En un principio, se identificaron porcentajes similares de testigos y víctimas, y un número menor de agresores a los reportados por otros estudios (Ávila-Toscano, Marenco-Escuderos y Tilano, 2014). El sexo, por su parte, aparece como una variable independiente del tipo de rol asumido por los estudiantes, hallándose de hecho, una distribución muy similar entre hombres y mujeres de acuerdo con los distintos roles. En este caso, a diferencia a trabajos previos (Cerezo et al., 2015), el sexo no resulta ser una variable significativa.
Se registró un alto número de estudiantes que asume el rol mixto agresor-víctima, tanto hombres como mujeres, lo que coincide con evidencias previas en el plano internacional (Del Rey y Ortega, 2008) y estudios con estudiantes colombianos (Ávila-Toscano et al., 2014; Ávila-Toscano, Osorio, Cuello, Cogollo y Hernández, 2010). De hecho, los investigadores han recalcado la necesidad de analizar atentamente las implicaciones psicosociales y emocionales de este conjunto de actores, en la medida que presentan mayores problemas de comportamiento y son a quienes sus compañeros perciben como los estudiantes con más dificultades relacionales (Cava, Musitu, Buelga y Murgui, 2010; Marenco-Escuderos y Tilano, 2013).

Precisamente, este grupo de actores fue el que mayor cantidad de diferencias significativas expresó en las estrategias empleadas por ambos progenitores; frente a la socialización parental muestran mayores niveles de estrategias basadas en la coerción/imposición por parte de la figura materna. Este grupo de estudiantes, más que los restantes roles, percibe en sus madres niveles elevados de formas de socialización basadas en la indiferencia, lo que supone escasa sensibilidad ante los hijos, falta de reconocimiento de sus conductas positivas y baja expresión de afecto. Asimismo, en los 
agresores-víctimas, la coerción verbal y la coerción física de las madres se reportan significativamente mayores que en los otros roles.

Los padres por su parte, al igual que las madres de los agresores-víctimas suelen ser indiferentes, lo que sugiere que pese a aceptar que sus hijos tienen conductas incorrectas, no establecen límites ni correcciones pertinentes. Asimismo, los padres recurren al uso del castigo físico con víctimas y agresores, mientras que con los testigos es notable la indiferencia. Los estudios enfocados en el análisis de estas variables coinciden en que las formas de corrección basadas en la punición y en la aplicación de correcciones severas mediadas por la violencia, parecen ser elementos que predisponen al hostigamiento entre iguales (Georgiou et al., 2013), así como a otro tipo de problemas en la adaptación psicosocial de las personas. El trato agresivo, o incluso el descuido o desinterés, son formas de crianza que pueden afectar la conducta de los menores; De hecho, incluso en adultos con historial de maltrato en la niñez se ha reportado que su estilo parental de crianza fue en esencia autoritario o negligente (Rikhye et al., 2008).

El segundo objetivo de este trabajo se enfocó en establecer si los roles asumidos por los estudiantes se relacionaban con el estilo de socialización de sus progenitores. Al respecto, vale decir que, a diferencia de otros estudios, los datos observados en este trabajo no muestran asociaciones tan claras entre los diferentes actores y el estilo de crianza de padre y madre. En los trabajos de Georgiou, (2008) y Cerezo et al. (2015), se ha reportado a la figura materna como sobreprotectora, esencialmente en relación con un estilo de educación permisivo o indulgente, lo que constituye un riesgo para la victimización en los estudiantes criados bajo este estilo parental. En efecto, en los estudiantes analizados en este estudio, la socialización materna sobresaliente es de tipo indulgente, lo que sugiere una crianza basada en la receptividad de las necesidades de los menores, pero con poco nivel de exigencia, por lo que se trataría entonces de madres con alta responsi- vidad, lo que coincide con evidencias previas (Charalampous et al., 2018). Sin embargo, a pesar de tratarse de la forma parental más repetida entre las madres, no se identifica una relación significativa a nivel estadístico con los roles asumidos por sus hijos. De hecho, en general, la evidencia obtenida en este estudio descarta que los diferentes estilos de socialización de la madre guarden relación con el rol o papel que asumen los estudiantes en las dinámicas de acoso.

Por su parte, en relación con la figura paterna, la crianza indulgente aparece como el único estilo asociado con los roles de acoso, puntualmente con el papel de testigo y agresor. En el primer caso, los datos obtenidos señalan que una mayor cantidad de testigos corresponde a estudiantes educados con un estilo paterno indulgente $(p=.00<.001)$, mientras que es menos común identificar estudiantes con el rol mixto de agresión y victimización con padres indulgentes $(p=.03<.05)$. La crianza responsiva, es decir, enfocada en atender demandas del menor con baja delimitación normativa, se reitera entre los padres, en quienes sobresale la indulgencia como el estilo de socialización parental más común, y que parece asociarse con la expectación del maltrato o intimidación sin que se realice intervención sobre dicho fenómeno. En este sentido, es compresible la relación negativa con el rol mixto de intimidación-victimización dado que, precisamente, este es el grupo de estudiantes con mayor implicación en las acciones violentas en la escuela en cuanto no solo agreden, sino que son receptores de agresiones (Del Rey y Ortega, 2008), papel contrapuesto al observador que se mantiene desobligado ante las acciones agresivas evidenciadas sin que por lo general se comprometan con la situación (Sullivan, Sullivan y Cleary, 2005).

A los padres indulgentes, les siguen en orden de frecuencia aquellos padres que muestran poco interés por la expresión de afecto, el seguimiento y el control normativo de sus hijos (padres negligentes). Entre los padres la orientación autoritativa se relega ante formas sociales 
de crianza que demandan poca exigencia y no están mediadas por el afecto positivo padrehijo. En oposición a estudios previos, el estilo autoritario en padres y madres, basado en la imposición de estrategias punitivas y el uso de la violencia verbal o psicológica como medio de resolución de problemas, fue el estilo de menor recurrencia, incluso no se halló relación entre el trato autoritario y el rol de agresor que ha sido señalada en trabajos anteriores (Gómez-Ortiz, 2016; López-Soler et al., 2009, Simões et al., 2015). Sin embargo, como se mencionó previamente, se trata de las formas más comunes de socialización empleadas por ambos progenitores.

Los resultados de esta investigación llevan a sostener, al igual que Cerezo et al. (2015), que no existe un estilo de crianza o una forma de interacción entre padres e hijos que defina con claridad los roles que asumen estos últimos en conductas de intimidación o victimización. En este estudio el aspecto más sobresaliente es el estilo indulgente de la figura paterna; sin embargo, el análisis de las pautas de interacción entre progenitores e hijos no se agota con el abordaje desarrollado en este estudio, sino que se debería encarar siendo conscientes de que se trata del reporte de la crianza percibida por los estudiantes. Nuevas indagaciones que apunten a la inclusión de otros factores de análisis resulta pertinente para los estudios futuros, considerando, por ejemplo, la participación del reporte de los propios padres, el abordaje de aspectos culturales (Georgiou et al., 2013; Georgiou et al., 2018), el reconocimiento de variables individuales que pueden participar en el proceso, entre otras consideraciones que sin duda ampliarían la discusión y el alcance del fenómeno.

Pese a estas limitantes, los resultados de este estudio muestran evidencia importante, pues por un lado, se reiteran las prácticas de acoso como un fenómeno real y repetitivo entre niños y adolescentes y, a su vez, recalcan la necesidad de asumir de forma crítica el papel de los actores que participan tanto de la intimidación como de la victimización (Del Rey y Ortega, 2008), atendiendo en este caso a las implicaciones sociofamiliares, pues como se ha visto, se trata de un conjunto amplísimo de estudiantes cuya crianza parece mediada por la coerción de sus padres y por la ausencia de vinculaciones afectivas sólidas provistas de afecto.

Por otro lado, este trabajo pone el acento sobre el importante rol que desempeñan las figuras parentales en la formación de habilidades psicosociales para la integración de niños y adolescentes, y llama la atención acerca de la forma en la que el padre y la madre se vinculan con sus hijos, en especial porque al comparar las estrategias de socialización entre los diferentes actores, las principales diferencias significativas recayeron sobre estrategias asociadas con la dimensión de coerción/imposición, o estrategias negativas de aceptación/ implicación como la displicencia o la indiferencia, sin que se dieran muestras significativas de afecto o diálogo, las cuales son estrategias generalmente relacionadas con una crianza basada en el apego y la delimitación de normas bien constituidas.

Esto demuestra la necesidad de trabajar por el desarrollo de la familia, y puntualmente por la construcción de relaciones paternofiliales basadas en estilos de crianza positivos, en los que se emplee la comunicación y se fijen normas precisas con un margen de supervisión o control normativo moderado, desde el cual se fomente la autonomía de los hijos y se estimule el crecimiento familiar por medio del apoyo y el afecto. Como señalan Gómez-Ortiz et al. (2016), este tipo de interacciones entre padres e hijos reemplaza el control autoritario excesivo y el uso de métodos disciplinarios coercitivos, sustituyendo así formas poco funcionales de interacción por valores personales indispensables para la vida.

\section{Referencias bibliográficas}

Andrade, J. y Gonz, J. (2017). Relación entre riesgo suicida, autoestima, desesperanza y estilos de socialización parental en estudiantes de bachi- 
1lerato. Psicogente, 20(37), 70-88. https://doi. org/10.17081/psico.20.37.2419

Ato, M., López, J. L. y Benavente, A. (2013). Un sistema de clasificación de los diseños de investigación en psicología. Anales de Psicología, 29(3), 1038-1059. https://doi.org/10.6018/ analesps.29.3.178511

Ávila-Toscano, J., Marenco-Escuderos, A. y Tilano, M. (2014). Redes de iguales y acoso escolar: evaluación desde el análisis de redes sociales. Psychologia: Avances de la Disciplina, 7(1), 53-64.

Ávila-Toscano, J. H., Osorio, L., Cuello, K., Cogollo, N. y Hernández, K. (2010). Conducta bullying y su relación con la edad, sexo y nivel de formación. Psicogente, 13(23), 13-26.

Baumrind, D. (1991). Parenting styles and adolescent development. En J. Brooks-Gunn, R. Lerner y A. Petersen (Eds.), The encyclopedia of adolescence (pp.746-758). New York: Garland.

Buelga, S., Cava, M. J. y Musitu, G. (2012). Reputación social, ajuste psicosocial y victimización entre adolescentes en el contexto escolar. Anales de Psicología, 28(1), 180-187.

Cava, M. J., Musitu, G., Buelga, S. y Murgui, S. (2010). The relationships of family and classroom environment with peer relational victimizations: an analysis of their gender differences. Spanish Journal of Psychology, 13(1), 156-165. https://doi.org/10.1017/S1138741600003747

Cerezo, F. y Ato, M. (2010). Social status, gender, classroom climate and bullying among adolescents pupils. Anales de Psicología, 26(1), 137-144.

Cerezo, F., Sánchez, C., Ruiz, C. y Arense, J. (2015). Roles en bullying de adolescentes y preadolescentes, y su relación con el clima social y los estilos educativos parentales. Revista de Psicodidáctica, 20(1), 139-155. https://doi. org/10.1387/RevPsicodidact.11097

Charalampous, K., Demetriou, C., Tricha, L., Ioannou, M., Georgiou, S., Nikiforou, M. y Stavrinides, P. (2018). The effect of parental style on bullying and cyberbullying behaviors and the mediating role of peer attachment relationships: A longitudinal study. Journal of Adolescence, 64(2), 109-123. https://doi. org/10.1016/j.adolescence.2018.02.003

Defensor del Pueblo. (2007). Violencia escolar: el maltrato entre iguales en la Educación Secundaria Obligatoria 1999-2006. Serie: Informes, estudios y documentos. Madrid: Esparme.

Del Rey, S. y Ortega, R. (2008). Bullying en los países pobres: prevalencia y coexistencia con otras formas de violencia. Internacional Journal of Psychology and Psychological Therapy, 8(1), 39-50.

Enríquez, M. y Garzón, F. (2015). El acoso escolar. Saber, ciencia y libertad, 10(1), 219-233.

Garaigordobil, M. y Machimbarrena, J. (2017). Stress, competence, and parental educational styles in victims and aggressors of bullying and cyberbullying. Psicothema, 29(3). 335-340. https://doi.org/10.7334/psicothema2016.258

García, F. y Gracia, E. (2010). ¿Qué estilo de socialización parental es el idóneo en España? Un estudio con niños y adolescentes de 10 a 14 años. Infancia y Aprendizaje, 33(3), 365-384. https://doi.org/10.1174/021037010792215118

Georgiou, S. N. (2008). Parental style and child bullying and victimization experiences at school. Social Psychology of Education, 11(3), 213-227. https://doi.org/10.1007/s11218-0079048-5

Georgiou, S. N., Ioannou, M. y Stavrinides, P. (2018). Cultural values as mediators between parenting styles and bullying behavior at school. Social Psychology of Education, 21(1), 27-50.

Georgiou, S. N., Stavrinides, P. y Fousiani, K. (2013). Authoritarian parenting, power distance, and bullying propensity. International Journal of School \& Educational Psychology, 1(3), 199-206.

Gómez-Ortiz, O., Del Rey, R., Casas, J. A. y Ortega-Ruiz, R. (2014). Estilos parentales e implicación en bullying. Cultura y Educación, 26(1), 132-158.

Gómez-Ortiz, O., Romera, E. y Ortega-Ruiz, R. (2016). Parenting styles and bullying. The mediating role of parental psychological aggression and physical punishment. Child Abuse \& Neglect, 51, 132-143. http://dx.doi. 
org/10.1016/j.chiabu.2015.10.025

Hoyos, H. Aparicio, J. y Córdoba, P. (2005). Caracterización del maltrato entre iguales en una muestra de colegios de Barranquilla (Colombia). Psicología desde el Caribe, 16, 1-28.

Landazabal, M. G. y Oñederra, J. A. (2010). Inteligencia emocional en las víctimas de acoso escolar y en los agresores. European Journal of Education and Psychology, 3(2), 243-256.

León del Barco, B., Felipe-Castaño, E., Polo del Río, M. y Fajardo-Bullón, F. (2015). Aceptación-rechazo parental y perfiles de victimización y agresión en situaciones de bullying. Anales de Psicología, 31(2), 600-606. http:// dx.doi.org/10.6018/analesps.31.2.156391

López-Soler, C., Puerto, J. C., Pina, J. A. L. y Prieto, M. (2009). Percepción de los estilos educativos parentales e inadaptación en menores pediátricos. Anales de Psicología, 25(1), 70-77.

Maccoby, E. E. y Martin, J. A. (1983). Socialization in the context of the family: Parent-child interaction. En P. H., Mussen (Ed.), Handbook of child psychology (pp. 1-101). New York: Wiley. Marenco-Escuderos, A. y Tilano, M. A. (2013). Estudiantes implicados en conductas de acoso. Un análisis de las características de conformación de sus redes en la escuela. En J. Ávila-Toscano (Comp.), Individuo, comunidad y salud mental. Avances en estudios sociales y aplicados a la salud (pp. 21-39). Barranquilla, Colombia: Ediciones UniReformada.

Musitu, G. y García, F. (2001). Escala de socialización parental en la adolescencia. Madrid: TEA.

Musitu, G. y García, J. F. (2004). Consecuencias de la socialización familiar en la cultura española. Psicothema, 16(2), 288-293.

Palomar, J. y Victorio, A. (2018). Predictores y correlatos del comportamiento prosocial de adolescentes mexicanos. Interdisciplinaria, 35(2), 495-509. https://doi.org/10.16888/ interd.2018.35.2.15

Parra, A. y Oliva, A. (2006). Un análisis longitudinal sobre las dimensiones relevantes del estilo parental durante la adolescencia. Infancia $y$ Aprendizaje, 29(4), 453-470.
Pérez, E. y Castañeda, I. (2015). El impacto de los estilos parentales en la dinámica de bullying a nivel secundaria. Revista Intercontinental de Psicología y Educación, 17(2), 77-101.

Resett, S. (2018). Estabilidad de ser victimizado, ser agresor, problemas emocionales y de conductas en adolescentes: ¿estabilidad o cambio? Interdisciplinaria, 35(2), 341-362. https://doi. org/10.16888/interd.2018.35.2.6

Rigby, K. (2005). Why do some children bully at school? The contributions of negative attitudes towards victims and the perceived expectations of friends, parents and teachers. School Psychology International, 26(2), 147-161.

Rikhye, K., Tyrka, A., Kelly, M., Gagne, G., Mello, A., Mello, M. (...) Carpenter, L. (2008). Interplay between childhood maltreatment, parental bonding, and gender effects: Impact on quality of life. Child Abuse \& Neglect, 32(1), 19-34. https://doi.org/10.1016/j.chiabu.2007.04.012

Salamanca-Ramos, E., Chávez-Ávila, P. y Carmona-Parra, J.A. (2017). Percepción de la autoridad parental en adolescentes escolarizados víctimas de desplazamiento. Aquichán, 17(4), 437-447. https://doi.org/10.5294/aqui.2017.17.4.7

Sánchez, C. (2009). Nivel de implicación en bullying entre escolares de educación primaria. Relación con el estatus sociométrico y la percepción del clima social familiar y escolar (Tesis doctoral). Universidad de Murcia. https:// bit.ly/2XMJXbm

Simões, S., Ferreira, J., Bbraga, S. y Testa, E. (2015). Bullying, vinculação e estilos educativos parentaisem adolescentes do $3^{\circ}$ ciclo do ensino básico. Revista Portuguesa de Investigação Comportamental e Social, 1(1), 30-41.

Smith, P. K. Morita, Y. Junger-Tas, J. Olweus, D. Catalana, R. y Slee, P. (1999). The nature of bullying. A cross national perspective. London: Routledge.

Sullivan, K., Sullivan, G. y Clearly, M. (2005). Bullying en la enseñanza secundaria: el acoso escolar, cómo se presenta y cómo afrontarlo. Barcelona: Ediciones CEAC.

Recibido: 26 de junio de 2019 Aceptado: 9 de noviembre de 2020 
